\section{Results of conservative management for consecutive esotropia after intermittent exotropia surgery}

\begin{abstract}
Purpose To examine the clinical course of consecutive esotropia (ET) using conservative management, after intermittent exotropia (IXT) surgery.

Methods This study included 149 out of 151 consecutive patients with ET after IXT surgery, who were managed conservatively. The clinical course of consecutive ET was examined and the patients were classified into two groups based on the duration of esodeviation: (1) $>3$ weeks (persistent ET group, $n=56$ ) and (2) $<3$ weeks (transient ET group, $n=93$ ). Patient characteristics and treatment outcomes, including the recurrence of exotropia and stereopsis, were compared between the two groups.
\end{abstract}

Results All patients with ET were managed with full-time alternate occlusion and/or with a Fresnel prism. In 149 patients out of 151 consecutive patients, $82 \%$ of ET disappeared at 12-month follow-up and all at the last follow-

Department of Ophthalmology, Kim's Eye Hospital, Konyang University College of Medicine, Seoul, Korea

${ }^{1}$ DW Kim and S Han are joint first authors.

Correspondence: S-H Baek, Department of Ophthalmology, Kim's Eye Hospital, Konyang University College of Medicine, Youngdeungpo-dong 4th 156, Youngdeungpo-gu, Seoul 150-034, Korea Tel: +82 226390811 ; Fax: +82 226779214 E-mail: drslitlamp@ kimeye.com

Received: 14 February 2014 Accepted in revised form: 7 January 2015

Published online:

27 March 2015 up visit (31.4 \pm 23.5 months). At the final visit, a recurrence of exotropia of $>10$ prism dioptres was significantly less frequent in the persistent ET group than in the transient ET group ( $25 \%$ vs $62 \%$, respectively; $P=0.01$ ). However, stereopsis outcome was not significantly different between the two groups, and stereopsis change was not affected by age. Conclusions By using conservative management only, persistent consecutive ET after IXT surgery disappeared in most cases by the 1-year follow-up visit after surgery. Recurrence of exotropia was significantly less frequent in patients with persistent ET, yet the sensory outcome was not affected by the duration of consecutive ET or age.

Eye (2015) 29, 776-782; doi:10.1038/eye.2015.16; published online 27 March 2015

\section{Introduction}

Consecutive esotropia (ET) immediately after the surgical management of intermittent exotropia
(IXT) is considered to be desirable because of a tendency toward postoperative exotropic drift; however, recent studies suggest that the longterm outcome of initial overcorrection is not necessarily positive. ${ }^{1,2}$ If consecutive ET persists, a patient may develop cosmetic problems and binocular vision dysfunction (eg, diplopia, suppression, or amblyopia). ${ }^{3-5}$ The initial management of consecutive ET can be nonsurgical (such as watchful waiting, alternate occlusion, or prism). If the ET persists $>6$ months after nonsurgical treatments, surgical treatment can be considered. However, the outcome of the surgical treatment of consecutive ET is reported to be variable..$^{3,6-8}$ The risks of reoperation and anesthesia, and the unpredictable long-term results of surgical treatment may be regarded to favor nonsurgical management for consecutive ET.

To the best of our knowledge, there are few reports in the English literature concerning the long-term clinical course of nonsurgical management of consecutive ET. Transient consecutive ET can be regarded as a desirable condition after exotropia surgery and may be associated with better alignment and with a small risk of developing binocular sensory dysfunction. This study investigated the clinical course of conservative management for persistent consecutive ET, which persisted $>3$ weeks after IXT surgery, and compared the results of persistent consecutive ET cases with transient ET cases, which persisted $<3$ weeks after surgery.

\section{Material and methods}

The study conformed to the medical protocol requirements of the Declaration of Helsinki, and the Institutional Review Board of Kim's Eye Hospital (Seoul, Korea) approved this study. Between January 2004 and July 2007, one surgeon (SHB) performed surgery for 
exodeviation in 850 patients. Patients who showed esodeviation at distance or at near after horizontal recti muscle surgery for IXT were identified and their records were reviewed. The indication of surgery for IXT included markedly noticeable exodeviation, usually of $\geq 20$ PD at distant fixation, with poor control. Patients were excluded if they had prior strabismus surgery, surgeries involving three or more horizontal rectus muscles or oblique muscles, constant exodeviation at distance and at near fixation, sensory exotropia following unilateral visual impairment, limitation in extraocular movement, or neurologic disorders. The following data were collected by reviewing the medical records: the patient's age, sex, visual acuity, refractive errors, presence of amblyopia or anisometropia, type of strabismus, preoperative and postoperative measurements of the near and distance deviation, the type and amount of surgery, and the results of the Titmus stereotest.

A comprehensive ophthalmic examination was performed in every patient before surgery. The examination included strabismus measurements by the prism and cover test and cycloplegic refraction. Sensory function was evaluated by using the Titmus stereoacuity test. The presence of amblyopia was defined as the difference of two or more lines in the Snellen visual acuity charts between the bestcorrected visual acuity of each eye or as a best-corrected visual acuity of 20/30 or lower.

All surgeries were performed under general anesthesia by one surgeon. The operative amount was determined on the basis of a standard table by Marshall Parks with modification. All patients received postoperative followup examinations, usually at 3 days, 1 week, 3 weeks, 3 months, 6 months, 12 months, and then every 6 months thereafter. At each follow-up examination, the ocular alignments at the distant and near distance fixations were recorded.

If the immediate postoperative ET, usually measured 1-2 days after surgery, persisted 2-3 weeks after the operation, alternating full-time patching was prescribed until the ET disappeared at distance and near fixations. If the ET did not improve sufficiently with alternate patching 1 or 2 months after surgery, base-out Fresnel press-on prisms (3 M Health Care, St Paul, Minneapolis, USA) were prescribed. When the patient needed to wear prisms for several months, regular spectacles

incorporated with a prism or prisms divided to each eye were prescribed. Bifocal correction was applied if patients showed orthophoria or small esophoria at distance and a larger ET angle for near vision.

Patients with postoperative esodeviation were classified into two groups, according to the ET duration: (1)

$>3$ weeks (ie, the persistent ET group) and (2) 3 weeks or less (ie, the transient ET group). In the analysis of the course of postoperative esodeviation, survival curves, and median survival time of esodeviation were evaluated for all patients (ie, for both groups) and for the persistent ET group patients only. Consecutive ET was defined as ET that appeared at near or distance fixation anytime during the follow-up period after the surgery. For survival analysis, the event was defined as the disappearance of esodeviation at both near and distance fixation. Clinical characteristics and potential factors that may influence differences in the clinical courses were compared between the persistent ET and transient ET groups. Recurrence of exotropia was determined when exodeviations of 10 or more prism dioptres (PD) at near fixation and at distance fixation developed during the follow-up period. The result of conservative management was evaluated by comparing the recurrence rate and stereopsis between the two groups during the follow-up period.

Effect of persistent overcorrection can be different by the age of the subjects. Loss of binocular function (loss of stereopsis and amblyopia development) was analyzed and compared between the transient ET and persistent ET groups stratified by age; younger stratum (7 years or younger) and older stratum (over 7 years).

The Kaplan-Meier method was used for survival analysis. The $\chi^{2}$-test, Fisher's exact test, and Student's $t$-test were used to compare variables between the two groups. Probability values $<0.05$ were considered statistically significant. All analyses were performed with IBM SPSS, version 20 software (SPSS Inc., Chicago, IL, USA).

\section{Results}

In this study, 151 patients were identified to have esodeviation at distance or at near after they had undergone horizontal muscle surgery only for IXT during the study period. One hundred and forty nine patients out of 151 consecutive patients with ET after IXT surgery $(98.7 \%)$ were followed with conservative management. Two patients underwent operation for persistent consecutive ET after conservative treatment. All patients were managed with full-time alternate occlusion or with a Fresnel prism or with both, usually starting 2-3 weeks after the IXT surgery. The mean age of the 149 patients was $7.2 \pm 2.7$ years, and men constituted $37 \%$ of all patients. The preoperative exodeviation was $27.9 \pm 5.9 \mathrm{PD}$ (range, 14-50 PD) at distance fixation and 25.3 $\pm 8.2 \mathrm{PD}$ (range, 0-50 PD) at near fixation. The mean follow-up time was $31.4 \pm 23.5$ months (range, 1-91 months). There were 56 patients in the persistent ET group and 93 in the transient ET group. In the persistent ET group, the number of children 7 years or younger (younger stratum) was $28(50 \%)$, and the number over 7 years (older stratum) was also 28 (50\%). In the transient ET group, the number of children in younger stratum was 51 
$(54.8 \%)$, and in older stratum was $42(45.2 \%)$. There was no significant difference in the age distribution (younger stratum vs older stratum) between persistent ET and transient ET groups $\left(P=0.393, \chi^{2}\right.$-test). In the persistent ET group, 26 (46\%) patients were managed with Fresnel prism glasses for a mean period of 12.1 months, after undergoing full-time alternate occlusion lasting for a mean period of 2.8 months.

According to the Kaplan-Meier survival analysis, the median survival time of esodeviation was 0.75 months in all ET patients and was 6 months in the persistent ET group (Figure 1). In the persistent ET group $(n=56)$, the proportion of patients with consecutive ET at 6 months was 41\% (19 patients), $18 \%$ (7 patients) at 12 months, and $0 \%$ (0 patients) at the final follow-up $(30.8 \pm 22.0$ months). This indicates that, after conservative treatment, most patients with persistent consecutive ET can experience the disappearance of esodeviation with a longer follow-up period. Among the 151 patients with consecutive esodeviation as a whole, esodeviation completely disappeared in 149 patients $(149 / 151=98.7 \%)$ by the last follow-up visit (31.4 \pm 23.5 months) with conservative management only.

A comparison of the persistent and transient ET groups showed no significant differences with regard to age at surgery, sex ratio, preoperative refractive error, preoperative stereopsis, and proportions of preoperative amblyopia or anisometropia. With respect to the type of IXT and the type of surgery, there also was no significant difference between the persistent and transient ET groups ( $P=0.64$ and $P=0.63$, respectively). However, the amount of immediate postoperative esodeviation was significantly different between the groups; the persistent ET group had a larger esodeviation at near fixation and at distance fixation than those of the transient ET group $(P=0.04$ and $P<0.001$, respectively; Table 1$)$. At

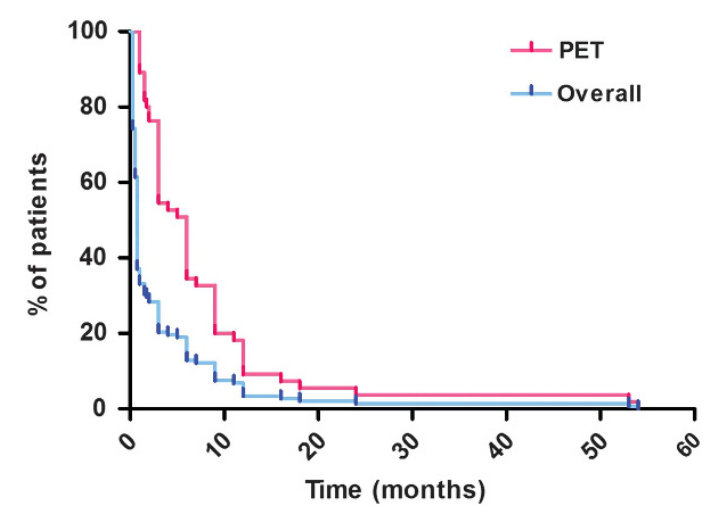

Figure 1 Kaplan-Meier survival curves of the persistent esotropia (ET) group (ie, consecutive ET lasting more than 3 weeks) and overall ET cases (ie, persistent and transient ET groups). The survival curves indicate that the estimated median survival times were 0.75 months for overall ET cases and 6 months for the persistent ET group. PET, persistent esotropia.
12 months after the IXT surgery and at the last follow-up examination, the rate of exotropia recurrence was significantly lower in the persistent ET group than in the transient ET group $(P<0.001$ and $P=0.01$, respectively; Table 2). The sensory outcome after conservative management showed that stereopsis was not significantly different between the two groups at 12 months after surgery and at the last follow-up examination $(P=0.71$ and $P=0.88$, respectively; Table 3 ).

A scatterplot of preoperative stereopsis and postoperative final stereopsis in persistent ET patients and transient ET patients stratified by age (age $\leq 7$ years and age $>7$ years) is shown in Figure 2. When a change of $\geq 2$ octaves of stereoacuity threshold was considered significant, there was only one patient with persistent ET in older age stratum at the final follow-up whose stereopsis deteriorated, and no significant difference in the distribution of stereopsis change (improvement/no change/deterioration) between the younger and older age strata either in the transient ET and persistent ET groups at the final follow-up visit $\left(P=0.745\right.$ by $\chi^{2}$-test). Overall, 19 patients had amblyopia preoperatively, and among them, 16 were in younger age stratum, the rest were over 7 years of age. Their BCVA at the final follow-up were improved and maintained within the 20/30 20/20 range, regardless of age and the duration of consecutive ET. There was no newly developed amblyopia after surgery regardless of age in either ET group.

\section{Discussion}

In this study, we demonstrated that most cases of consecutive ET after IXT surgery could be improved with nonsurgical management only and that, with this conservative management scenario, the outcome of IXT surgery was better in the persistent ET group than in the transient ET group with regard to the recurrence of exotropia. We found that in 149 of 151 consecutive patients with ET after IXT surgery $(149 / 151=98.7 \%)$ who were followed with conservative management, $82 \%$ $(51 / 62=82.3 \%)$ of ET disappeared after 12 months follow-up and all, $100 \%$, at the last follow-up visit $(31.4 \pm 23.5$ months). The results of this study are comparable to those of previous studies of nonsurgical management, even though, the patient characteristics and the definition of consecutive esotropia were different. Hardesty $^{9}$ and Veronneau-Troutman ${ }^{10}$ reported five and eight cases of consecutive esotropia, respectively, treated with base-out prism. They showed that prism glasses were helpful to establish fusion and to reduce the angle of esodevation for some patients without additional surgery. Lee and Hwang ${ }^{11}$ also reported that prismatic correction after weeks of alternating full-time patching helps to maintain motor and sensory outcomes for a long time in 
Table 1 Comparison of the patient groups based on the duration of esotropia

\begin{tabular}{|c|c|c|c|}
\hline & Persistent ET $(\mathrm{n}=56)$ & Transient ET $(\mathrm{n}=93)$ & P-value \\
\hline Age at IXT surgery $($ mean $\pm S D)$ & $7.6 \pm 3.0$ & $6.9 \pm 2.2$ & 0.13 \\
\hline Men, no. $(\%)$ & $16(28.6 \%)$ & $39(41.9 \%)$ & 0.12 \\
\hline \multicolumn{4}{|l|}{ Spherical equivalent (mean $D \pm S D$ ) } \\
\hline OD & $-1.34 \pm 2.04$ & $-1.05 \pm 1.85$ & 0.37 \\
\hline OS & $-1.39 \pm 2.03$ & $-1.01 \pm 1.61$ & 0.22 \\
\hline \multicolumn{4}{|l|}{ Associated feature, no. (\%) } \\
\hline Amblyopia & $9(16 \%)$ & $10(11 \%)$ & 0.55 \\
\hline Anisometropia & $0(0 \%)$ & $6(7 \%)$ & 0.08 \\
\hline \multicolumn{4}{|c|}{ Preoperative deviation, mean $P D \pm S D$ (range) } \\
\hline Distance & $27.7 \pm 6.0(18-40)$ & $28.0 \pm 5.8(14-50)$ & 0.25 \\
\hline Near & $25.1 \pm 8.7(0-40)$ & $26.1 \pm 8.1(10-50)$ & 0.20 \\
\hline \multicolumn{4}{|c|}{ Immediate postoperative esodeviation, mean $P D \pm S D$ (range) } \\
\hline Distance & $11.1 \pm 7.6(8-40)$ & $8.6 \pm 6.3(10-40)$ & 0.04 \\
\hline Near & $10.2 \pm 8.7(8-30)$ & $5.5 \pm 6.8(10-40)$ & $<0.001$ \\
\hline Follow-up, months \pm SD (range) & $30.8 \pm 22.0(1-84)$ & $31.8 \pm 24.53(2-91)$ & 0.82 \\
\hline Exotropia pattern, no. (\%) & & & 0.64 \\
\hline Basic & $49(88 \%)$ & $82(88 \%)$ & \\
\hline Convergence insufficiency & $1(2 \%)$ & $0(0 \%)$ & \\
\hline Divergence excess & $1(2 \%)$ & $2(2 \%)$ & \\
\hline Pseudodivergence excess & $5(9 \%)$ & $9(10 \%)$ & \\
\hline Type of surgery, no. (\%) & & & 0.63 \\
\hline $\mathrm{R}$ and $\mathrm{R}$ & $54(97 \%)$ & $91(98 \%)$ & \\
\hline BLR recession & $2(3 \%)$ & $2(2 \%)$ & \\
\hline
\end{tabular}

Abbreviations: BLR recession, bilateral lateral rectus muscle recession; D, dioptre; ET, esotropia; PD, prism dioptre; R and R, lateral rectus muscle recession and medial rectus muscle resection.

Table 2 Recurrence rates of exotropia in patient groups based on the duration of esodeviation

\begin{tabular}{lccc}
\hline Recurrence & $\begin{array}{c}\text { Persistent ET } \\
(\mathrm{n}=56)\end{array}$ & $\begin{array}{c}\text { Transient ET } \\
(\mathrm{n}=93)\end{array}$ & P-value $^{\text {a }}$ \\
\hline 12 months & $6(11 \%)$ & $46(50 \%)$ & $<0.001$ \\
Final FU & $14(25 \%)$ & $58(62 \%)$ & 0.01 \\
\hline
\end{tabular}

Abbreviations: ET, esotropia; FU, follow-up; XT, exotropia.

${ }^{\mathrm{a}}$ On the basis of the $\chi^{2}$-test.

patients with consecutive esotropia. On the other hand, Dawson et al. ${ }^{12}$ reported that 19 of 60 (28.3\%), which included 15 of $36(41.7 \%)$ in a fusional potential group, obtained and maintained remarkable ocular alignment with a single injection of Botulinum toxin in surgically overcorrected exotropia. Botulinum toxin injection to the medial rectus is reported to be an effective treatment for persistent overcorrection after exotropia surgery.

However, in South Korea, Botulinum toxin injection is not covered by the national health insurance; therefore it is not frequently used as the primary treatment modality for strabismus, whereas occlusion and/or prism are widely utilized. To the best of our knowledge, here we additionally report a long-term follow-up survival analysis of consecutive ET using nonsurgical management after exotropia surgery in a largest consecutive ET population ever in which nearly all patients underwent conservative management only.

Immediate reoperation is considered when there is restriction in the movement of the lateral rectus muscle on the operated eye, thereby causing ET. Surgical treatment for consecutive ET is otherwise generally considered for patients with persistent consecutive ET that is $\geq 14$ PD at distance, for patients showing an increased angle of esodeviation, or for patients with a limitation in lateral gaze at 6 months after surgery. ${ }^{13}$ In this study, the median survival time of ET was 6 months for patients with persistent ET lasting > 3 weeks. Among the 151 patients, two patients underwent operation for consecutive ET at 12 weeks and 18 weeks after IXT surgery. Each preoperative esodeviation of the two patients was $25 \mathrm{PD}$, 25 PD at distance fixation and 25 PD, 35PD at near fixation. Two patients showed limitation in extraocular movement at lateral gaze. There is a poor compliance of patients with full-time alternate occlusion. Also, we identified four patients with postoperative ET of $>14$ PD at 6 months. Using only conservative management, all esodeviations in these cases disappeared by 15 months after the initial exotropia surgery; therefore, ET surgery was unnecessary. 
Table 3 Comparison of the sensory outcomes in patient groups based on the duration of esodeviation

\begin{tabular}{lccc}
\hline Stereopsis & $\begin{array}{c}\text { Persistent ET } \\
(\mathrm{n}=56)\end{array}$ & $\begin{array}{c}\text { Transient ET } \\
(\mathrm{n}=93)\end{array}$ & P-value \\
\hline Preoperation & & & \\
$\quad$ Log arcsec (mean $\pm \mathrm{SD})$ & $1.94 \pm 0.27$ & $1.90 \pm 0.28$ & 0.55 \\
$\quad$ Mean arcsec & 87.1 & 79.4 & \\
Postoperative & & & 0.71 \\
12 months & & & \\
Log arcsec (mean $\pm \mathrm{SD})$ & $1.84 \pm 0.25$ & $1.82 \pm 0.20$ & \\
$\quad$ Mean arcsec & 69.2 & 66.1 & 0.88 \\
Final follow-up & & & \\
$\quad$ Log arcsec (mean $\pm \mathrm{SD})$ & $1.82 \pm 0.23$ & $1.81 \pm 0.24$ & 64.6 \\
$\quad$ Mean arcsec & 66.1 & &
\end{tabular}

Abbreviation: ET, esotropia.

${ }^{a}$ On the basis of Student $t$-test.

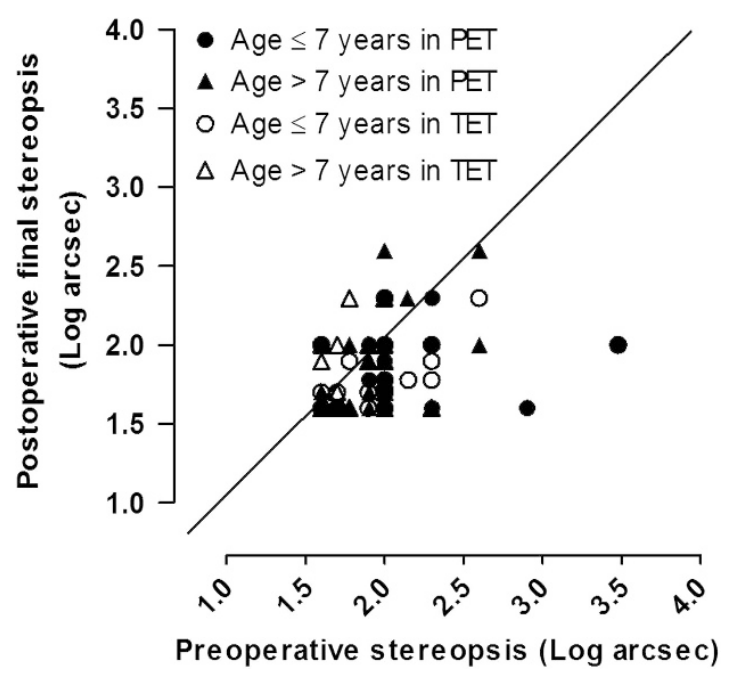

Figure 2 Scatterplot showing the relationship between preoperative stereopsis and postoperative final stereopsis in persistent ET patients and transient ET patients stratified by age (age $\leq 7$ years and age $>7$ years). PET, persistent esotropia; TET, transient esotropia.

We set the end point of consecutive ET to the time by which all esodeviations disappeared at distance and near fixation, and did not differentiate esophoria with good stereopsis and esotropia with diplopia, because we found that the alteration patterns of esodeviation and diplopia are variable and clear distinction between esophoria with single vision and esotropia with diplopia was not always possible nor constant when we review records of our patients. The patients with persistent overcorrection manifested diplopia by Worth 4 Dot test in $10 \sim 20 \%$ and proportions of subjects with suppression by Worth 4 Dot test decreased whereas those of good Titmus stereopsis increased proportional to their follow-up periods. (data not shown).

Consecutive ET is defined as the conversion from exodeviation to esodeviation as a result of overcorrection of exotropia that is not associated with external mechanical factors or with acquired paretic factors. While a consecutive esotropia may resolve spontaneously over time, it was reported that consecutive esotropia may persist in $6-20 \%$ as persistent overcorrection, ${ }^{14-16}$ and the rate of persistent overcorrection can be differ by the definition of the condition and study population. In the present study, consecutive ET was defined as ET appearing at near or distance fixation anytime during the follow-up period after the surgery and developed in 151 $(18 \%)$ of 850 patients who underwent exotropia surgery, and the rate of persistent overcorrection that lasted more than 3 weeks was calculated to be $6.6 \%(=56 / 850$ total exodeviation surgery by one surgeon during the study period). However, there are possibilities of persistent overcorrection among the 699 patients excluded from the study, therefore, the rate of persistent overcorrection was believed to be greater than $6.6 \%$ in this population.

Exodrift usually occurs after exotropia surgery; therefore, overcorrection is considered satisfactory immediately after exotropia surgery. Paik and $\mathrm{Cho}^{17}$ advised 6-10 PD esodeviation immediately after lateral rectus recession in both eyes; Lee et al. ${ }^{18}$ advised 11-20 PD esodeviation; and Carlos and Carlos ${ }^{19}$ advised 10 PD esodeviation. In this study, the persistent ET group showed a significantly larger immediate postoperative esodeviation at near and distance fixation and a lower long-term exotropia recurrence rate than the transient ET group. In the persistent ET patients, the binocular visual function improved in comparison with the preoperative function (mean stereopsis, 87.1 arcsec vs 66.1 arcsec, respectively). The postoperative stereopsis was not statistically different between the persistent ET and the transient ET groups. The aim of strabismus surgery is to achieve good binocular visual function and to improve cosmetic appearance. Without worse visual function compromise, a serious disadvantage of consecutive ET, this study supports the need for overcorrection in exotropia surgery to decrease the recurrence rate of exotropia.

The recurrence rate after surgery for IXT has been known to vary, because of the large variation in inclusion and exclusion criteria, length of follow-up, and definition of success. Pratt-Johnson et $a l^{20}$ found that $59 \%$ of 100 cases of IXT recurred after a primary operation, compared with $5 \%$ of 111 cases by Richard and Parks, ${ }^{21}$ and $40 \%$ of 67 cases (over 10 PD at near and distance fixation, mean follow-up of 3.3 years) by Beneish and Flanders. ${ }^{22}$ In this study, the transient ET group demonstrated $62 \%$ of recurrence rate after IXT surgery with mean follow-up period of $31.8 \pm 24.53$ (range 2-91) months, although we could not estimate the recurrence rate of the whole-IXT surgery population including subjects who did not show any esotropia after surgery. Nonetheless, this recurrence rate in our transient ET group is rather high, and this 
might be related to the relatively low persistent ET rate (6.6\% or higher) in our population.

Surgical methods may influence exotropia surgery results such as consecutive ET. Different exotropia types using the Duane and Burian classification may exhibit different clinical courses. There are few reports about the incidence of consecutive ET after each type of surgery and few studies report that the surgical methods influence the development of consecutive ET. Keech and Stewart ${ }^{23}$ report that the consecutive ET rate was 7.1\% after monocular recession and resection procedures, $8.5 \%$ after bilateral lateral rectus recession, and $37.5 \%$ after surgery involving three or four horizontal rectus muscles. Lee and Hwang ${ }^{11}$ reported $11.2 \%$ (44 of 392 patients who underwent bilateral lateral rectus recession), 54.5\% (115 of 211 patients who underwent unilaateral resection and recession), and $12.5 \%$ (7 of 56 patients who underwent unilateral lateral rectus recession) of persistent overcorrection, respectively, when defined as continued esodeviation of 5 PD (at distance fixation) of more for 4 weeks after exotropia surgery. In the present study, surgeries involving three or more horizontal rectus muscles were excluded. The number of pseudodivergence excess cases and the number of bilateral lateral rectus recession surgeries were small. We could not determine a meaningful relation between the incidence of consecutive ET and the type of surgery.

Effect of persistent overcorrection can be different by the age of the subjects, and younger subjects are prone to loss of binocular function than older subjects. When we analyzed and compared loss of stereopsis and amblyopia development between the transient ET and persistent ET groups stratified by age of 7 years, no significant difference was found between the younger and older age strata either in the transient ET and persistent ET groups. This might be the result of conservative management we used to prevent the development of suppression or amblyopia or both, to our study population of simple IXT with good preoperative stereopsis (87.1 arcsec in persistent ET group, 79.4 arcsec in transient ET group). Good preoperative stereopsis may be associated with good surgical results ${ }^{22}$ and poor preoperative stereopsis is reportedly associated with more frequent or longer consecutive ET. Lew et al ${ }^{13}$ report that consecutive ET occurs more frequently in patients that have stereopsis worse than 3000 arcsec. In the current study, preoperative stereopsis was not significantly different between the transient and the persistent ET groups, and there was no significant difference between the stereopsis test results of the two groups at the 1-year visit and the final follow-up visit. In addition, none of the sex, preoperative refractive error, or anisometropia influenced the persistence of consecutive ET in this study.

Suppression, decreased visual acuity, and amblyopia in visually premature children can develop because of consecutive ET. ${ }^{4,24}$ These outcomes usually occur in cases of persistent ET. Various frequencies of amblyopia due to consecutive ET have been reported. Kim and Hwang ${ }^{25}$ reported 2 consecutive ET patients (from 68 patients) who developed amblyopia; Kim and $\mathrm{Choi}^{8}$ reported a newly developed case of amblyopia in 1 of 18 ET patients; and Keech and Stewart ${ }^{23}$ reported no cases of amblyopia among all of their patients who were over 8 years old. In this studies, we could not identify any cases of newly developed amblyopia in the persistent ET group regardless of their age, when $50 \%$ of them were in amblyogenic age, 7 years or younger. This may be due to our conservative management. With the reports from other studies, we believe that if we carry out proper conservative management, occurrence of amblyopia would be rare in consecutive esodeviation after IXT surgery.

A limitation of this study is its retrospective design. The compliance of treatments, that is patching or prism glasses wearing, was not thoroughly monitored. However, it is clinically important that 149 of 151 consecutive ET patients improved with only conservative treatment. This finding supports the favorable role of conservative management of consecutive ET without significant limitation in extraocular movement, rather than early surgical intervention, for managing consecutive ET patients.

In conclusion, with conservative management, most cases of persistent consecutive ET disappeared at distance and at near fixation by 1 year after IXT surgery. Also, on the last follow-up visit ( $31.4 \pm 23.5$ months), 149 patients of the 151 patients with consecutive esodeviation could be improved with nonsurgical management only. After the conservative management of persistent ET, the recurrence of exotropia was significantly less frequent and the sensory outcome was not affected by the duration of ET. There was no significant difference in loss of binocular function (loss of stereopsis and amblyopia development) between the younger and older age strata either in the transient ET and persistent ET groups in this study population, and this may be the result of conservative management.

\section{Summary}

What was known before

- If the ET persists more than 6 months after nonsurgical treatments, surgical treatment can be considered. However, the outcome of the surgical treatment of consecutive ET is variable.

What this study adds

- Consecutive ET disappeared in 149 out of 151 patients, mostly within 1 year after intermittent exotropia surgery, with conservative management only.

- Recurrence of exotropia was significantly less frequent in patients with persistent ET, yet the sensory outcome was not affected by the duration of consecutive ET. 


\section{Conflict of interest}

The authors declare no conflict of interest.

\section{References}

1 Buck D, Powell CJ, Sloper JJ, Taylor R, Tiffin P, Clarke MP et al. Surgical intervention in childhood intermittent exotropia: current practice and clinical outcomes from an observational cohort study. Br J Ophthalmol 2012; 96: 1291-1295.

2 Leow PL, Ko ST, Wu PK, Chan CW. Exotropic drift and ocular alignment after surgical correction for intermittent exotropia. J Pediatr Ophthalmol Strabismus 2010; 47: 12-16.

3 Shin YJ, Chang BL. The clinical outcome of the consecutive esotropia after surgical correction. J Korean Ophthalmol Soc 2003; 44: 2085-2090.

4 Edelman PM, Brown MH, Murphree AL, Wright KW. Consecutive esodeviation. Then what? Am Orthopt J 1988; 38: 111-116.

5 Von noorden GK. Binocular Vision and Ocular Motility. Mosby: MA, 1996, pp 352-355.

6 Son AN, Park SC, Lee WR. Clinical study of consecutive esotropia. J Korean Ophthalmol Soc 1990; 31: 1328-1334.

7 Kim SJ, Lee SH, Woo KH. Surgery for correcting the consecutive esotropia. J Korean Ophthalmol Soc 1994; 35: 332-336.

8 Kim YH, Choi MY. The effect of Fresnel prism treatment in consecutive esotropia. J Korean Ophthalmol Soc 2006; 47: 1623-1629.

9 Hardesty HH. Treatment of under and overcorrected intermittent exotropia with prism glasses. Am Orthopt J 1969; 19: 110-119.

10 Véronneau-Troutman S. Prism adaptation test (PAT) in the surgical management of acquired esotropia. Arch Ophthalmol 1991; 109: 765-766.

11 Lee EK, Hwang JM. Prismatic correction of consecutive esotropia in children after a unilateral recession and resection procedure. Ophthalmology 2013; 120: 504-511.

12 Dawson E L, Marshman W E, Lee J P. Role of Botulinum toxin A in surgically overcorrected exotropia. J AAPOS 1999; 3: 269-271.
13 Lew H, Lee JB, Han SH, Park HS. Clinical evaluation on the consecutive esotropia after exotropia surgery. J Korean Ophthalmol Soc 1999; 40: 3482-3490.

14 Burian HM, Spivey BE. The surgical management of exodeviations. Am J Ophthalmol 1965; 59: 603-620.

15 Dunlap EA. Overcorrection in horizontal strabismus surgery. In: New Orleans Academy of Ophthalmology Symposium on Strabismus. Mosby: St. Louis, 1971, pp 255-267.

16 Hardesty HH, Bonyton JR, Keenan JP. Treatment of intermittent exotropia. Arch Ophthalmol 1978; 96: 268-274.

17 Paik HJ, Cho YA. Recession of the lateral recti in intermittent exotropia evaluation of the amount of immediate postoperative deviation. J Korean Ophthalmol Soc 1990; 31: 1445-1450.

18 Lee JH, Lee SY, Lee YC. The effect of lateral rectus muscle advancement in consecutive esotropia after bilateral rectus muscle recession. J Korean Ophthalmol Soc 2008; 49: 1801-1806.

19 Carlos SD, Carlos FU. Postoperative evolution of the planned initial overcorrection in intermittent exotropia: 61 cases. Binocular Vision Eye Muscle Surg Q 1993; 8: 141-148.

20 Pratt-Johnson JA, Barlow JM, Tilson G. Early surgery in intermittent exotropia. Am J Ophthalmol 1977; 84: 689-694.

21 Richard JM, Parks MM. Intermittent exotropia: Surgical results in different age groups. Ophthalmology 1983; 90: 1172-1177.

22 Beneish R, Flanders M. The role of stereopsis and early postoperative alignment in long-term results of intermittent exotropia. Can J Ophthalmol 1994; 29: 119-124.

23 Keech RV, Stewart SA. The surgical overcorrection of intermittent exotropia. J Pediatr Ophthalmol Strabismus 1990; 27: 218-220.

24 Raab EL. Difficult esotropia entities: principles of management. In: Rosenbaum AL, Santiago AP (eds). Clinical Strabismus Management. WB Saunders: Philadelphia, 1999, pp 149-150.

25 Kim TW, Kim JH, Hwang JM. Long-term outcome of patients with large overcorrection following surgery for exotropia. Ophthalmologica 2005; 219: 237-242. 